\author{
NATIONAL PROGRAMME FOR ANALYSIS OF ON-'THE-FARM \\ SOW RECORDS \\ - Utilization of results by the farmer \\ - Analysis of factors of productivity
}

\title{
National computerized programme for analysis of on-the-farm sow records
}

\author{
J. DAGORN \\ Institut technique du Porc, \\ 149, rue de Bercy, \\ 75579 Cedex 12
}

The National computerized programme for analysis of on-the-farm sow records was elaborated by the following organisms : U. N. M. I., I. T. P. and I. N. R. A. and started in 1970. The purpose is the following :

- individual utilization of the programme : supply of accurate informations to the farmer about the mean performances of his herd and the situation of the latter as compared to a reference group ;

- collective utilization : establishment of a "data bank" from the informations collected allowing estimation of the real efficiency of new animal production techniques.

On October ist 1974, this programme included 3250 farmers, among which $83 \mathrm{p}$. Ioo were members of an association of animal producers. Within two years, the increase in the numerical productivity of the sows was about I piglet per sow and per year, while the size of the herds increased by 12 sows.

On july Ist 1974, an analysis of data from 1386 farms was made, revealing the variations in the analytical criteria according to the numerical productivity (table $\mathrm{I}$ ). 


\begin{tabular}{|c|c|c|c|c|c|}
\hline \multirow{14}{*}{ 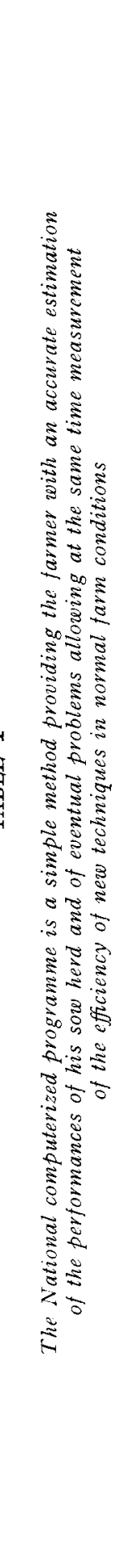 } & 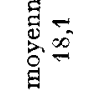 & & $\bar{i}$ & $\begin{array}{l}\text { : } \\
\text { - }\end{array}$ & $\begin{array}{l}\infty_{\alpha} \\
\infty \\
\infty\end{array}$ \\
\hline & 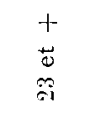 & $\stackrel{\infty}{-\infty}$ & $\hat{\stackrel{s}{O}}$ & $\stackrel{8}{=}$ & 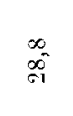 \\
\hline & 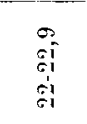 & 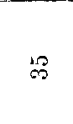 & in & $\stackrel{3}{=}$ & $\stackrel{0}{\stackrel{\circ}{\circ}}$ \\
\hline & $\frac{0}{\vec{i}}$ & $\stackrel{S}{S}$ & $\stackrel{+3}{s}$ & 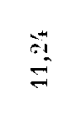 & 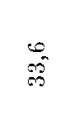 \\
\hline & 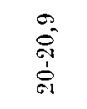 & 点 & $\begin{array}{l}\infty \\
\stackrel{+}{+}\end{array}$ & $\stackrel{\ominus}{=}$ & $\begin{array}{l}0 \\
\text { is } \\
\text { in }\end{array}$ \\
\hline & $\begin{array}{l}\sigma \\
\stackrel{5}{\sigma} \\
\dot{\sigma}\end{array}$ & 令 & $\vec{\Xi}$ & $\begin{array}{l}\hat{B} \\
\hat{\Xi}\end{array}$ & $\overrightarrow{\mathrm{s}}$ \\
\hline & $\begin{array}{l}\vec{x} \\
\vec{\infty} \\
\vec{\infty}\end{array}$ & $\overline{\vec{O}}$ & 象 & $\begin{array}{l}\hat{i} \\
\stackrel{1}{\Xi}\end{array}$ & $\bar{\Xi}$ \\
\hline & $\begin{array}{l}8 \\
: \\
5\end{array}$ & $\frac{a}{a v}$ & $\stackrel{r}{31}$ & $\begin{array}{l}\overline{0} \\
\stackrel{0}{E}\end{array}$ & $\begin{array}{l}\infty \\
\infty \\
\infty\end{array}$ \\
\hline & $\begin{array}{l}0 \\
\dot{0} \\
\dot{0}\end{array}$ & $\underset{\sigma}{ت}$ & ڤi & $\hat{\leftrightarrow}$ & $\stackrel{1}{=}$ \\
\hline & 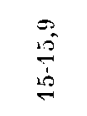 & 8 & 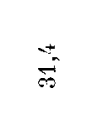 & 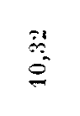 & $\stackrel{+}{*}$ \\
\hline & 吾 & iD & $\therefore$ & $\stackrel{20}{=}$ & $\stackrel{8}{:}$ \\
\hline & $\begin{array}{l}\overrightarrow{9} \\
\hat{\sigma}\end{array}$ & 落 & 8 & $\begin{array}{l}\stackrel{D}{\infty} \\
0 \\
\infty\end{array}$ & 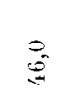 \\
\hline & $\stackrel{2}{1}$ & $\hat{i}$ & $\stackrel{n}{\stackrel{n}{0}}$ & $\stackrel{5}{2}$ & $\stackrel{0}{=}$ \\
\hline & 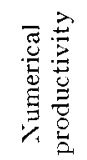 & 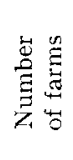 & 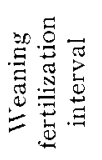 & 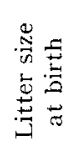 & 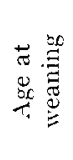 \\
\hline
\end{tabular}

